heterogeneity of dying processes. Therefore, this study presents a longitudinal mixed modeling approach to analyze terminal drop of affective well-being, treating time-to-death as right-censored variable to include the study survivors, and an exploratory analysis of potential heterogeneity of timeto-death-related patterns. Depressive symptoms (CES-D) from the Longitudinal Aging Study Amsterdam ( $N=3107$, 6 measurement occasions, 3-year intervals) were analyzed. The findings confirm a crucial terminal drop dynamic even when those who survived the observation period are included. Moreover, the analyses revealed evidence of considerable heterogeneity of time-to-death-related trajectories, in terms of amount and shape of terminal change in depressive symptomatology.

\section{DYADIC ASSOCIATIONS IN WELL-BEING IN THE LAST YEARS OF LIFE}

G. Hülür ${ }^{1}$, F.J. Infurna ${ }^{2}$, N. Ram ${ }^{3}$, D. Gerstorf ${ }^{1}, 1$.

Humboldt University, Berlin, Germany, 2. University of Arizona, Tempe, Arizona, 3. Pennsylvania State University, University Park, Pennsylvania

Research on terminal decline has generally focused on how the last years of life are being experienced individually. Here, we take a different approach by studying how wellbeing changes in couples as one partner approaches death. Specifically, we examine how well-being trajectories of the surviving partner are associated with the well-being trajectories of the dying partner. We used annual longitudinal data obtained over 30 years in the German Socio-Economic Panel from 2,156 couples with 20,513 longitudinal observations. Results revealed that late-life well-being trajectories were indeed linked within couples: Yearly well-being fluctuations of the surviving partner were closely associated with yearly well-being fluctuations of the dying partner. The strength of such within-couple links differed between partners and follow-up analyses will target the contributing role of individual and partnership variables. We discuss factors that contribute to within-couple well-being associations and the role of close social relationships in the last years of life.

\section{TERMINAL DROP OF WELL-BEING IN VERY OLD}

AGE

M. Wettstein, O.K. Schilling, Institute of Psychology,

Department of Psychological Ageing Research, University of Heidelberg, Heidelberg, Germany

Those who survive into oldest-old age may expect and have developed resilience towards terminal processes, hence showing less terminal decline in well-being than evidenced with samples with a broader range of age-at-death. The present study analyzed time-to-death-related change in a broad range of facets of well-being (affective, eudaimonic, death-related fears), making use of longitudinal oldest-old data ( $N=125$, aged $87-96$ at baseline) that provides up to 18 measurements with short (3-6 month) intervals, hence enabling observation of changes in close proximity to death. Confirming our expectations, terminal drop did not unfold de-differentiated across all facets of well-being, but appeared pronounced only for indicators of positive affect. Further analyses suggest that this terminal drop was driven by loss of functional abilities. Altogether, the findings point at the oldest-olds' resilience towards terminal experiences - except the interruption of positive affect due to terminal loss of behavioral competences which are needed for prohedonic activities.

\section{AGE-RELATED AND DEATH-RELATED DIFFERENCES IN EMOTIONAL COMPLEXITY}

Y. Palgi ${ }^{1}$, A. Shrira ${ }^{2}$, M. Ben-Ezra ${ }^{3}$, T. Spalter ${ }^{4}$, G. Kave ${ }^{5}$, D. Shmotkin 6 , 1. University of Haifa, Haifa, Israel, 2.

Bar-Ilan University, Ramat Gan, Israel, 3. Ariel University, Ariel, Israel, 4. University of Toronto, Toronto, Ontario, Canada, 5. The Open University, Raanana, Israel, 6. Tel Aviv University, Tel Aviv, Israel

Emotional complexity, as seen in covariation between retrospective judgments of positive and negative affects, has shown mixed evidence. We propose that emotional complexity may remain intact or even increase in old age, and yet it decreases in light of functional deterioration shortly before death.

We used 3 large-scale databases: 2 cross-sectional (SHARE, $N=17,437, M_{\text {age }}=64$; HRS, $N=6,032, M_{\text {age }}=67$ ) and 1 longitudinal (CALAS, $N=1,310, \mathrm{M}_{\text {age }}=83$ ). Hierarchical multiple regressions and multilevel models showed that respondents who perceived themselves as closer to death or were actually closer to death showed lower emotional complexity (a stronger negative correlation between positive and negative affects). Age and emotional complexity were unrelated or positively related, depending on the sample. The results indicate that both subjective and objective closeness to death are associated with lower emotional complexity. This death-related decrease in emotional complexity is discussed within current theories of aging.

\section{SESSION 4160 (SYMPOSIUM)}

\section{UNDERSTANDING ALCOHOL USE IN OLDER ADULTHOOD: PSYCHOSOCIAL CORRELATES AND CONSEQUENCES}

Chair: P. Sacco, University of Maryland, Baltimore, Maryland

Discussant: A. Kuerbis, Hunter College/CUNY, New York, New York

Alcohol is the most commonly used substance among adults aged 65 and older, and the prevalence of alcohol consumption is likely to increase in coming years as the babyboom generation reaches older adulthood. Even though alcohol use ubiquitous among older adults, aging research specific to alcohol use is limited. In particular, limited research has evaluated factors that drive consumption among older adults as well as psychosocial correlates of use. This symposium will discuss psychological and social factors associated with alcohol use including qualitative data on use, the role of self-medication as a motive for drinking, the relationship of alcohol consumption and elder abuse and alcohol use in long term care settings.

Dr. Haighton will review research focused on self-identified reasons for drinking among older adults, and will discuss her qualitative research directed at understanding reasons for drinking among older adults in the United Kingdom. Using Dr. Haighton's work as a foundation, Drs. Canham and Mauro will delve specifically into the self-medication 